\title{
Prendre soin des personnes après AVC : réactions émotionnelles des aidants informels hommes et femmes
}

\author{
Caring for a stroke victim: the emotional reactions \\ of male and female caregivers
}

Barbara Bucki (1), Elisabeth Spitz ${ }^{(2)}$, Michèle Baumann (3)

\begin{abstract}
Résumé : Deux ans après la survenue d'un accident vasculaire cérébral (AVC), les réactions émotionnelles des aidants informels hommes et femmes et leurs répercussions sociales et psychologiques ont été analysées. 62 dyades patient (64,4 ans) / aidant informel (59,3 ans) ont répondu à domicile à un questionnaire administré par un enquêteur: 1) déficiences résiduelles de l'AVC classées selon l'American Heart Association Stroke Outcome Classification ; 2) réactions émotionnelles du 'Caregiver Reaction Assessment'; 3) évolution de la répartition des activités au sein du foyer familial. Les retombées de l'aide affectent davantage les femmes que les hommes sur l'état de santé, l'organisation de l'emploi du temps et le manque de soutien familial. Les femmes se sentent "fatiguées » $(50 \%$ vs $12,5 \%$ des hommes), ne parviennent pas à " trouver du temps pour se détendre " $(35,7 \%$ vs $6,3 \%)$ et estiment que "les autres se sont déchargés sur elles » (35,7\% vs 11,8\%). Plus qu'avant la survenue de l'AVC, elles estiment assurer les responsabilités au sein du foyer $(37,5 \%$ vs $5,9 \%$ ). En revanche, les hommes déclarent "prendre plaisir à s'occuper» des patient-e-s (93,8\% vs $67,9 \%$ chez les femmes) et organiser plus qu'avant des loisirs tels que les vacances ( $29 \%$ vs $5,6 \%$ ) et les invitations d'amis (20,6\% vs $10,5 \%)$. L'épuisement des aidants, en particulier des femmes, les définit aujourd'hui comme une "population à risque ". Des interventions comme le trialogue et le counseling devraient permettre aux professionnels de mieux répondre à leurs besoins.
\end{abstract}

Mots-clés : Soutien informel - accident cérébrovasculaire - femme - homme.

Summary: The objective of this study was to assess the emotional response to caregiving among stroke caregivers and to assess the social and psychological impact of strokes on households two years after the event. Questionnaires were administered to 62 patient (64.4 years old)/caregiver (59.3 years old) dyads. The survey focused on: 1) residual impairments, based on the American Heart Association Stroke Outcome Classification; 2) the Caregiver Reaction Assessment (CRA - 24 items - 5 dimensions); and 3) changes in the division of household tasks (seven items). Although male and female caregivers care for patients with similar residual impairments, the study found that female caregivers were more likely to be affected by the impact of caregiving on their health and schedule and by lack of family support. Women were more likely to feel "tired all the time" (50\% vs. 12.5\%), to struggle to "find time to relax" (35.7\% vs. 6.3\%) and to feel that "others dumped caring onto them" (35.7\% vs. $11.8 \%$ ). Since the onset of stroke, women had also taken on more responsibilities in the household (37.5\% vs. 5.9\%), while men were more likely to "enjoy caring" for patients (93.8\% vs. 67.9\%) and to have become more involved in organizing holidays (29\% vs. 5.6\%) and seeing friends (20.6\% vs. $10.5 \%)$. Because of the high level of exhaustion, particularly among women, caregivers are a high-risk population for the healthcare system. Interventions such as trialogue and counseling are required to meet the needs of male and female caregivers.

Keywords: Caregivers - stroke - women - men.

(1) Doctorante PhD sciences sociales PhD psychologie - Cotutelle de thèse entre Unité Recherche INSIDE université du Luxembourg - L-7201 Walferdange - Luxembourg et EA4360 APEMAC Université de Nancy - Université Paul Verlaine Metz - Université Paris Descartes, EPSaM, 57000 Metz - France.

(2) Professeure psychologie de la santé - EA4360 APEMAC Université de Lorraine - Université Paris Descartes, EPSaM, 57000 Metz - France.

(3) Professeure sociologie de la santé responsable du projet AVC financé par le Fonds National de la Recherche VIVRE/06/06/06 - Unité Recherche INSIDE Université du Luxembourg - Walferdange - Luxembourg. 


\section{Introduction}

Au Luxembourg comme dans les pays de l'Union Européenne, les accidents vasculaires cérébraux (AVC) représentent la première cause de handicap acquis à l'âge adulte et la deuxième cause d'invalidité d'origine neurologique [1]. L'AVC est un événement de vie majeur dont la survenue entraîne des bouleversements sur les plans économique et psychologique, mais aussi familial et social: changements dans le partage des tâches et dans les activités telles que les sorties, loisirs, activités culturelles, et dans la vie relationnelle/sociale avec les amis, en particulier dans le couple lorsque les séquelles touchent la sphère intime [2].

La diminution des finances publiques, et plus récemment l'application du principe de responsabilisation des familles à l'égard de la santé, ont contribué à réduire les durées de séjour en milieu hospitalier [3]. De nombreux soins sont dorénavant prodigués à domicile par les membres des familles appelés " aidants informels ", " aidants naturels » ou encore " aidants familiaux ", qui jouent un véritable rôle social et économique [4]. Bien que le sentiment envers le parent ou l'ami soit un facteur primordial de leur motivation, la grande majorité des aidants (conjoints, enfants, parents ou amis) n'a pas eu le choix d'assumer ce rôle, celui-ci leur étant plus ou moins imposé par la maladie de leur proche. Le but de notre étude est de comprendre comment les aidants informels pourraient être mieux accompagnés car cette question d'actualité concerne directement, ou est susceptible de concerner dans un avenir proche, la plus grande partie d'entre nous.

La continuité des soins à apporter quotidiennement à des personnes dont les séquelles touchent tant les déficiences motrices que neurologiques ou psycho-comportementales et la disponibilité nécessaire pour réaliser les tâches qui en découlent constituent pour les aidants informels un fardeau [3]. Cette charge de travail, qu'elle soit objective ou même subjective [5], est associée à une satisfaction des conjoints des victimes d"AVC inférieure à la population générale, à l'égard de la vie, de leurs activités quotidiennes, des loisirs et contacts sociaux et de leur vie sexuelle et sentimentale avec leur partenaire [6]. Réduction de leur champ d'indépendance, diminution de leurs activités de loisirs et temps passé à prodiguer des soins, les responsabilités nouvelles apparaissent comme des sources de stress [7], d'anxiété [8], de souffrances psychologiques [9] et de dépression [10], d'autant plus si la santé des aidants informels était déjà fragile avant l'événement [11]. La fréquence de la dépression est plus élevée chez les aidants que chez les patients, notamment s'ils vivent en couple avec la victime d'AVC[12]. Quant aux membres des familles, ils modifient leur manière de résoudre les problèmes, de communiquer, de s'impliquer affectivement et de définir leurs rôles [13].

\section{Vécu social et psychologique des femmes et des hommes aidants informels}

$\mathrm{Au}$ sein des familles, "l'aide à autrui » est encore perçue comme l'extension d'un rôle altruiste traditionnellement dévolu aux femmes [14]. Les aidants informels restent majoritairement féminins [15], bien que la proportion d'hommes qui assistent à domicile un(e) proche malade soit en 
constante augmentation [16]. Les observations empiriques de distinctions entre femmes et hommes dans leur manière de prendre soin de l'autre et dans leurs réactions psychologiques, sociales et émotionnelles ont amené les chercheurs à en analyser les impacts. Ainsi, les femmes semblent apporter une aide plus complexe [11]. Cependant, lorsqu'elles travaillent, ce sont également elles qui éprouvent plus de difficultés à concilier leur rôle de personne-soutien avec leur vie professionnelle [2], de telle sorte que leurs relations familiales [13] et leurs activités quotidiennes [6] en sont perturbées, et qu'elles se sentent submergées par leurs responsabilités familiales [17]. Elles sont davantage anxieuses [9] et développent, en tant qu'épouse, une vigilance autour de leur conjoint AVC [18].

Toutefois, au sein du foyer familial et en fonction de la gravité de l'AVC, le rôle des hommes aidants informels est amené à se modifier [19]. Réaliser des tâches domestiques, intégrer des gestes associés à l'aide, dispenser des soins corporels à leurs épouses deviennent pour certains des actes quotidiens [20]. D’ailleurs, les aidants hommes déclarent éprouver un sentiment d'injustice sociale [2], mais reconnaissent que cet évènement a renforcé les liens familiaux [21].

La problématique de notre étude s'inscrit dans l'identification et la compréhension, deux ans après un AVC, des différences de genre face à l'aide informelle. Nous nous sommes intéressés aux compétences psychosociales présentes chez les aidants informels, en interaction avec leur contexte culturel et environnemental au travers de travaux qui s'appuient sur le concept de " health capability » [22]. Ruger a tenté de modéliser l'atteinte ou le maintien potentiel de l'état de santé en tenant compte à la fois des capacités de l'individu à maintenir son état de santé, et sa liberté de faire des choix de santé. Ce concept donne autant de valeur à la réalisation des buts qu'à l'opportunité de les atteindre. Dans notre étude, l'intervalle de deux ans a été privilégié car il révèle les conséquences à long-terme subies par les aidants informels qui ont réorganisé leur quotidien, leurs repères et leurs rôles [23]. Reconsidérer la phase post chronique et développer des interventions en direction des familles et des patients font partie intégrante de la déclaration européenne d'Helsingborg des objectifs à atteindre pour 2015 [24] et des stratégies concernant les AVC; d'où la pertinence d'identifier afin de mieux comprendre les compétences psychosociales en jeu dans ces dyades familiales.

Notre objectif est de mesurer et de comparer, deux ans après la survenue d'un AVC, les réactions émotionnelles produites par l'aide informelle apportée par les aidants selon leur sexe aux patients AVC dont ils s'occupent, et d'évaluer la manière dont ils perçoivent l'évolution de leurs activités et responsabilités au sein de leurs foyers familiaux.

\section{Méthodologie}

\section{Population}

Les aidants informels ont été désignés par des victimes d'AVC participant une étude sur l'après AVC au Luxembourg, pour laquelle l'Inspection Générale de la Sécurité Sociale a identifié toutes les victimes d'un AVC de 
juillet 2006 à juin 2007 selon les codes ICD-10 correspondant à un AVC. Les critères d'inclusion des patients étaient les suivants : résider au Luxembourg au moment de l'AVC, avoir été pris en charge au Luxembourg, être en vie deux ans après l'AVC et être hébergé hors institution. Tous les critères, dont le diagnostic d'AVC, ont été vérifiés, après consentement des personnes éligibles a priori, sur la base des informations du dossier d'hospitalisation des patients.

Les 102 personnes volontaires ont été contactées par téléphone pour convenir d'un rendez-vous. À cette occasion, ils ont été invités à désigner leur aidant principal comme étant « la personne qui s'occupe le plus d'[eux/elles] depuis l'AVC".

\section{Méthode}

Les patients et les aidants ont été interrogés chacun par un(e) enquêteur(trice), en face à face, à leur domicile, dans la langue de leur choix (français, luxembourgeois, allemand ou portugais).

\section{Aspects éthiques}

Le protocole de l'étude a obtenu l'approbation du Comité National d'Éthique de la Recherche et a fait l'objet d'une déclaration au Comité de Protection des Données du Luxembourg.

\section{Instruments de recueil des données}

Deux questionnaires distincts ont été créés, incluant des échelles validées en français. Les versions luxembourgeoise, allemande et portugaises ont été traduites, contre-traduites puis relues par des traducteurs professionnels de langue maternelle.

- Contenu du questionnaire destiné aux personnes AVC :

- variables sociodémographiques : sexe, âge ;

- types de déficiences (motrices, visuelles, sensorielles, du langage, de la mémoire) tels que définis par l'American Heart Association Stroke Outcome Classification [25].

- Contenu du questionnaire destiné aux aidants :

- variables sociodémographiques: sexe (variable dépendante), lien de parenté avec la personne AVC, situation professionnelle au moment de l'AVC et au moment de l'enquête ;

- réactions face à l'accompagnement, mesurées par le «Caregiver Reaction Assessment " (CRA-24 items) [26], conçu pour apprécier les réactions positives et négatives des personnes qui assistent un malade au quotidien. L'échelle explore cinq dimensions: estime de l'aide (7 items), manque de soutien familial ( 5 items), impact de l'aide sur les ressources financières ( 3 items), sur l'emploi du temps ( 5 items) et sur la santé (4 items). Les items ont été traduits et adaptés dans les quatre langues. Les modalités de réponse sont les suivantes: "tout à fait d'accord", "plutôt d'accord», "neutre", "plutôt pas d'accord" ou " pas du tout d'accord»; 
- tâches et responsabilités menées au sein du foyer familial, liste de sept variables établie à l'issue d'entretiens qualitatifs exploratoires [2], dont les modalités de réponse varient: " plus », " autant» ou "moins » qu'avant l'AVC.

\section{Analyses statistiques}

L'ensemble des variables a été étudié en fonction du sexe de l'aidant, en ajustant les résultats selon l'âge des aidants informels et leur niveau d'instruction. Étant donné que le CRA a été validé dans sa version française [3] mais pas dans les versions allemande, portugaise et luxembourgeoise utilisées dans notre enquête, nous avons ajouté à l'analyse par dimension une analyse par item. Les réponses attribuées aux 24 items du CRA ont été dichotomisées de la manière suivante : "Tout à fait/plutôt d'accord " vs " pas du tout/plutôt pas d'accord/neutre ». Pour les variables continues, la normalité de la distribution a été vérifiée pour chaque sexe par le test de Kolmogorov-Smirnov puis la force des liens a été calculée à l'aide du test $t$ de Student. Pour les variables catégorielles, nous avons utilisé le test du chi² de Wald. Le seuil de significativité a été fixé à 0,1.

\section{Résultats}

Au total, 62 dyades victimes d'AVC / aidants informels ont participé à l'étude.

\section{Caractéristiques sociodémographiques et sanitaires des personnes AVC (tableau I)}

Les 62 patients AVC dont les aidants prennent soin se composent de 25 femmes (40,3\%) et 37 hommes $(59,7 \%)$ âgés de 64,4 ans $(\sigma=15,8)$. Ceux qui ont désigné un aidant de sexe féminin sont plus âgés et plus fréquemment cadres supérieurs ou professionnels indépendants que les personnes ayant désigné un aidant de sexe masculin. Deux ans après l'AVC, les patients gardent au moins une déficience sensorielle $(53,2 \%)$, souffrent de troubles de la mémoire $(38,7 \%)$, du langage $(35,5 \%)$, de déficiences motrices $(33,9 \%)$ et visuelles $(22,6 \%)$. Si les aidantes féminines s'occupent plus fréquemment que les hommes de personnes qui présentent des troubles de la mémoire, la fréquence des autres séquelles est similaire, quel que soit le sexe de l'aidant.

\section{Caractéristiques sociodémographiques des aidants informels (tableau II)}

Le profil regroupe 40 femmes (65,5\%) âgées en moyenne de 59,1 ans $(=13,9)$ et 22 hommes $(34,4 \%)$ âgés de 59,6 ans $(=13,9)$. Les aidants ont en moyenne 5,1 ans de moins que les victimes d'AVC dont ils s'occupent et $48,4 \%$ sont âgés de 60 ans et plus. La majorité est le conjoint des personnes AVC (51 couples), quel que soit le sexe de l'aidant.

La situation professionnelle des aidants diffère selon le genre : les hommes sont plus nombreux à travailler et les femmes sont plus nombreuses à être sans activité professionnelle. La situation professionnelle des aidants reste 
Tableau I : Caractéristiques sociodémographiques et sanitaires des personnes atteintes d'un AVC selon le sexe de l'aidant principal

\begin{tabular}{|c|c|c|c|c|}
\hline & \multirow{2}{*}{$\begin{array}{c}\text { Total des } \\
\text { personnes } \\
\text { atteintes } \\
\text { d'AVC } \\
N=62\end{array}$} & \multicolumn{2}{|c|}{$\begin{array}{l}\text { Personnes atteintes d'AVC } \\
\text { ayant désigné un aidant : }\end{array}$} & \\
\hline & & $\begin{array}{l}\text { Féminin } \\
N=40\end{array}$ & $\begin{array}{l}\text { Masculin } \\
N=22\end{array}$ & \\
\hline & $m(\sigma)$ & $m(\sigma)$ & $m(\sigma)$ & $p$ \\
\hline Âge & $64,4(15,8)$ & $67,9(14,2)$ & $59,4(15,7)$ & $0,037^{*}$ \\
\hline \multirow[t]{2}{*}{$\begin{array}{l}\text { Nombre de domaines affectés } 2 \text { ans } \\
\text { après l'AVC }[0 ; 5]\end{array}$} & $2,2(1,8)$ & $2,5(2,0)$ & $2,6(1,8)$ & 0,865 \\
\hline & $\%$ & $\%$ & $\%$ & $p$ \\
\hline Féminin & 40,3 & 10,0 & 95,5 & \\
\hline Masculin & 59,7 & 90,0 & 04,5 & \\
\hline Profession & & & & $0,007^{\star *}$ \\
\hline N'a jamais travaillé & 18,9 & 08,8 & 36,8 & \\
\hline Ouvrier & 22,6 & 29,4 & 10,5 & \\
\hline Employé, technicien & 35,8 & 29,4 & 47,4 & \\
\hline Cadre supérieur, indépendant & 22,6 & 32,4 & 05,3 & \\
\hline Niveau d'instruction & & & & 0,210 \\
\hline Inférieur à 12 ans d'études & 45,3 & 51,4 & 33,3 & \\
\hline 12 ans d'études ou supérieur & 54,7 & 48,6 & 66,7 & \\
\hline \multicolumn{5}{|l|}{ Déficiences 2 ans après l'AVC } \\
\hline Sensorielles & 53,2 & 60,0 & 40,9 & 0,188 \\
\hline De la mémoire & 38,7 & 47,5 & 22,7 & $0,064 \S$ \\
\hline Du langage & 35,5 & 40,0 & 27,3 & 0,409 \\
\hline Du langage & 33,9 & 40,0 & 22,7 & 0,262 \\
\hline Visuelles & 22,6 & 22,5 & 22,7 & 1,000 \\
\hline
\end{tabular}

(1) Niveau de signification du t de Student ou du $p<0,1 \S: p<0,05^{\star},<0,01^{\star \star}$.

semblable entre la survenue de l'AVC et deux ans après, seule la fréquence de la retraite ayant augmenté d'environ $6 \%$.

Les hommes aidants informels ont, plus fréquemment que les femmes, réalisé un minimum de 12 ans d'études, mais femmes et hommes sont majoritairement représentés dans la catégorie employé/technicien.

\section{Réactions des aidants face au fait d'assister un malade au quotidien}

\section{Analyse du Caregiver Reaction Assessment par dimension (tableau III)}

Parmi les cinq dimensions du Caregiver Reaction Assessment, trois présentent des différences selon le sexe de l'aidant. Les femmes rapportent un plus grand retentissement de l'aide sur l'organisation de leur temps $(p=0,004)$. Elles sont plus affectées par le manque de soutien familial $(p=0,024)$ et estiment qu'aider leur proche a un impact sur leur santé $(p=0,039)$.

L'estime accordée à l'aide informelle et l'impact de l'AVC sur les ressources financières sont perçus de manière semblable par les femmes et les hommes (respectivement $p=0,284$ et $p=0,755$ ). 
Tableau II : Caractéristiques sociodémographiques déclarées par les aidants informels $(n=62)$

\begin{tabular}{|c|c|c|c|c|}
\hline & $\begin{array}{c}\text { Total } \\
N=62\end{array}$ & $\begin{array}{c}\text { Aidants } \\
\text { féminins } \\
N=40\end{array}$ & $\begin{array}{c}\text { Aidants } \\
\text { masculins } \\
N=22\end{array}$ & \\
\hline & $m(\sigma)$ & $m(\sigma)$ & $m(\sigma)$ & $p$ \\
\hline \multirow[t]{2}{*}{ Âge } & $59,3(13,7)$ & $\begin{array}{c}59,1 \\
(13,9)\end{array}$ & $\begin{array}{c}59,6 \\
(13,9)\end{array}$ & 0,901 \\
\hline & $\%$ & $\%$ & $\%$ & $p$ \\
\hline Sexe & & 64,5 & 35,5 & \\
\hline Lien avec la personne AVC & & & & 0,329 \\
\hline Conjoint & 82,3 & 81,6 & 90,9 & \\
\hline Autre & 17,7 & 18,4 & 09,1 & \\
\hline Profession & & & & 0,083 \\
\hline N'a jamais travaillé & 17,5 & 27,0 & 00,0 & \\
\hline Ouvrier & 14,0 & 13,5 & 15,0 & \\
\hline Employé, technicien & 49,1 & 43,2 & 60,0 & \\
\hline Cadre supérieur, indépendant & 19,3 & 16,2 & 25,0 & \\
\hline Niveau d'études & & & & $0,047^{\star}$ \\
\hline Inférieur à 12 ans d'études & 42,4 & 51,3 & 25,0 & \\
\hline 12 ans d'études ou supérieur & 57,6 & 48,7 & 75,0 & \\
\hline Sit. pro. au moment de l'AVC & & & & $0,004^{\star \star}$ \\
\hline En activité & 40,4 & 32,4 & 55,0 & \\
\hline À la retraite & 26,3 & 18,9 & 40,0 & \\
\hline Sans activité & 33,3 & 48,6 & 05,0 & \\
\hline Sit. pro. 2 ans après l'AVC & & & & $0,049^{\star}$ \\
\hline En activité & 33,9 & 30,8 & 45,0 & \\
\hline À la retraite & 32,3 & 28,2 & 45,0 & \\
\hline Sans activité & 33,8 & 41,0 & 10,0 & \\
\hline
\end{tabular}

(1) Niveau de signification du $t$ de Student pour la variable continue âge, du test exact de Fischer pour la variable niveau d'instruction et du $\chi^{2}$ pour les autres variables : $p<0,05^{\star},<0,01^{\star \star}$.

Tableau III : Les domaines du Caregiver Reaction Assessment en fonction du genre

\begin{tabular}{|c|c|c|c|c|c|}
\hline \multirow[b]{2}{*}{ Domaines } & \multicolumn{2}{|c|}{$\begin{array}{l}\text { Aidants féminins } \\
\qquad N=40\end{array}$} & \multicolumn{2}{|c|}{$\begin{array}{c}\text { Aidants masculins } \\
\qquad N=22\end{array}$} & \multirow[b]{2}{*}{$p^{(2)}$} \\
\hline & $m^{1}$ & $\sigma$ & $m^{(1)}$ & $\sigma$ & \\
\hline Estime de l'aide & 73,0 & 19,4 & 78,8 & 9,7 & 0,284 \\
\hline Manque de soutien familial & 39,9 & 24,7 & 25,9 & 15,5 & $0,024^{*}$ \\
\hline Impact sur les finances & 21,1 & 21,5 & 19,6 & 15,3 & 0,755 \\
\hline Impact sur l'emploi du temps & 46,7 & 29,7 & 24,7 & 19,3 & $0,004^{\star \star}$ \\
\hline Impact sur la santé & 33,0 & 22,1 & 19,5 & 19,5 & $0,039^{*}$ \\
\hline
\end{tabular}

(1) $\mathrm{m}=$ moyennes $[0 ; 100] ; \sigma=$ écarts-types.

(2) Niveau de significativité obtenu en testant l'effet du sexe dans un modèle de régression linéaire simple, ajusté sur l'âge des aidants informels et leur niveau d'éducation (moins de 12 ans d'études vs. 12 ans et + ) : $p<0,05^{\star}, p<0,01^{\star \star}$. 


\section{Analyse du CRA par items (tableau IV)}

La moitié des femmes rapporte qu'elles se sentent "toujours fatiguées » contre seulement $12,5 \%$ des hommes $(p=0,022)$. Les femmes éprouvent plus fréquemment que les hommes des difficultés pour "trouver du temps pour [se] détendre à cause des interruptions fréquentes de [leurs] activités » $(p=0,040)$. Les hommes sont plus nombreux que les femmes à prendre du " plaisir à s'occuper» du/de la patient(e) AVC $(93,3 \%$ vs 67,9\%; $p=0,078)$.

La proportion de femmes qui estiment que "[leur] famille [les] laisse seules » pour aider la personne AVC $(44,4 \%)$ est supérieure à celle des hommes $(18,8 \% ; p=0,086)$.

\section{Évolution des tâches et des responsabilités (tableau $V$ )}

Depuis la survenue de l'AVC, les tâches réalisées moins souvent sont, pour les femmes, l'organisation des vacances (concerne $29 \%$ des femmes) et, tant pour les femmes que pour les hommes, la réalisation des invitations $(20,6 \%$ des femmes et $10,5 \%$ des hommes).

Les tâches réalisées plus souvent depuis l'AVC par les femmes sont l'organisation des rapports avec les professionnels (pour $52 \%$ d'entre elles) et les responsabilités en général dans le foyer (37,5\%). Chez les hommes, les activités qui ont augmenté en fréquence sont la conduite en voiture (concerne $21,1 \%$ d'entre eux) et les tâches ménagères, la cuisine et les courses $(15,8 \%)$.

La grande majorité des hommes estime que la réalisation des tâches est restée stable depuis l'AVC (entre $73,7 \%$ et $94,1 \%$ des réponses selon l'activité). En revanche, les proportions des femmes qui assurent plus qu'avant les rapports avec les professionnels ( $52 \%$ des femmes vs $6,2 \%$ des hommes; $p=0,023$ ) et dont les responsabilités au sein du foyer ont augmenté $(37,5 \%$ des femmes vs $5,9 \%$ des hommes; $p=0,065 \S)$ sont supérieures à celles des hommes.

\section{Discussion}

Les aidants informels qui accompagnent au domicile des patients AVC ont perçu des répercussions sociales et ont eu des réactions émotionnelles différentes selon leur genre. Les hommes sont plus nombreux à ne rapporter aucun changement dans leurs activités quotidiennes depuis l'AVC. Si la majorité des aidants, sexe confondu, perçoivent avoir accordé de l'estime aux activités d'aide menées, les femmes déclarent être plus affectées, tant sur le plan physique que dans l'organisation et l'évolution de leurs tâches et responsabilités.

D’après nos résultats, les aidants femmes et hommes semblent faire face à un fardeau relativement égal. Pourtant, l'impact sur l'emploi du temps est fortement souligné en fonction du genre, ce qui corrobore les résultats d'études antérieures $[17,27]$ et suggère que les femmes mettent fréquemment de côté les activités de leur vie quotidienne pour s'occuper de la personne AVC.

Dans notre étude, les femmes reconnaissent un manque de soutien familial, estiment que leurs familles les laissent seules s'occuper des 
Tableau IV : Pourcentages d'accords avec les items du Caregiver Reaction Assessment selon le genre

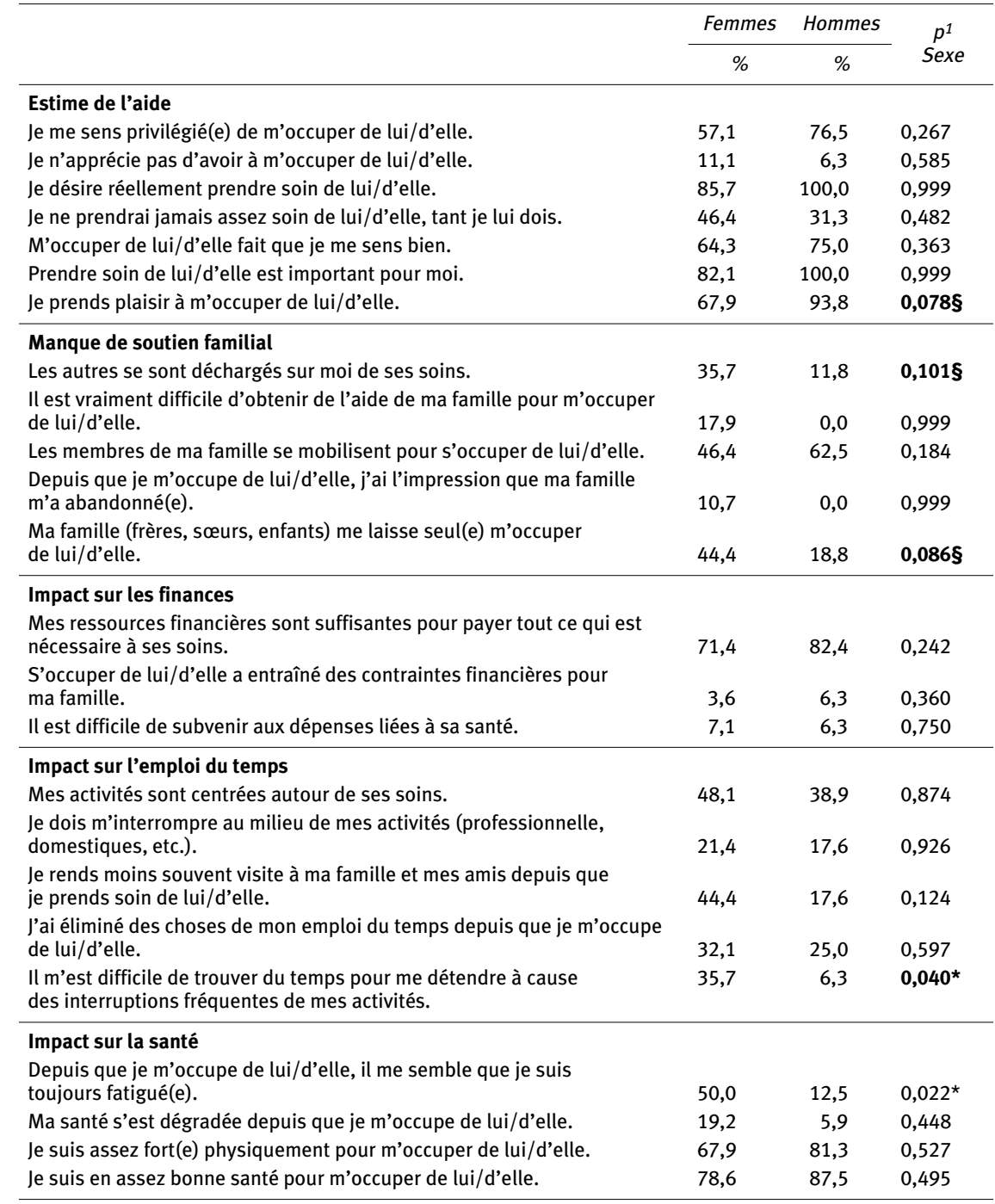

(1) Niveau de signification du $\chi^{2}$ de Wald en ajustant les résultats selon l'âge des aidants informels (variable continue) et leur niveau d'instruction ( $<12$ ans d'études $v s \geq 12$ ans) : $p<0,1 \S, p<0,05^{\star}$.

personnes AVC et qu'il est difficile pour elles de trouver de l'aide. Cette solitude, qui accroît inévitablement le fardeau des femmes aidantes, se retrouve chez les aidantes de personnes atteintes d'un cancer [28]. Nos résultats s'ancrent donc dans le spectre plus large des différences de genre observées dans l'aide informelle en général. À ce propos, une métaanalyse révèle entre autres que les femmes aidantes sont en moins bonne santé et 
Tableau V: Fréquence des déclarations concernant l'évolution des tâches et activités des aidants informels dans le foyer depuis l'AVC

\begin{tabular}{lcccccccc}
\hline & \multicolumn{3}{c}{ Femmes (\%) } & \multicolumn{5}{c}{ Hommes (\%) } \\
\cline { 2 - 9 } & - & $=$ & + & - & $=$ & + & $p^{(1)}$ \\
\hline $\begin{array}{l}\text { Je fais les tâches ménagères, la cuisine } \\
\text { les courses. }\end{array}$ & 11,8 & 76,4 & 11,8 & 5,3 & 78,9 & 15,8 & 0,888 \\
\hline Je fais les démarches administratives. & 2,9 & 79,4 & 17,7 & 5,0 & 85,0 & 10,0 & 0,545 \\
\hline J'organise les vacances, les loisirs. & $\mathbf{2 9 , 0}$ & 64,5 & 6,5 & 5,6 & 88,8 & 5,6 & 0,307 \\
\hline $\begin{array}{l}\text { Je fais les invitations d'amis et/ou } \\
\text { de la famille. }\end{array}$ & 20,6 & 70,6 & 8,8 & $\mathbf{1 0 , 5}$ & 89,5 & 0 & 0,951 \\
\hline $\begin{array}{l}\text { Je conduis la voiture. } \\
\text { J'assure les rapports avec les professionnels } \\
\text { (médecins, femmes de ménage, etc.) }\end{array}$ & $\mathbf{6 , 7}$ & 83,3 & 10,0 & 5,2 & 73,7 & $\mathbf{2 1 , 1}$ & 0,475 \\
\hline J'ai des responsabilités dans le foyer familial. & 0 & $\mathbf{4 0}$ & $\mathbf{5 2}$ & 0 & $\mathbf{9 3 , 8}$ & $\mathbf{6 , 2}$ & $\mathbf{0 , 0 2 3 *}$ \\
\hline
\end{tabular}

(1) Niveau de signification du $\chi^{2}$ de Wald, pour la réalisation d'un modèle linéaire généralisé ajustant le sexe des aidants sur leur âge et leur niveau d'instruction (moins de 12 ans d'étude vs 12 ans et + ) : $p<0,1 \S, p<0,05^{\star}$.

déclarent un bien-être inférieur aux hommes [29]. Ce résultat tend peut-être aussi à refléter la persistance du stéréotype selon lequel les femmes s'épanouissent en apportant leur aide à autrui[14]. Ayant intégré ce stéréotype, les femmes sont peut-être elles-mêmes amenées à mettre en place, face à leurs proches, des stratégies pour laisser paraître qu'elles n'ont pas besoin d'aide. Elles répondent ainsi aux attentes sociales liées à leur genre [30].

Dès lors, le niveau élevé d'estime qu'elles accordent aux activités d'aide pourrait être envisagé en termes d'accommodation à l'environnement familial et social. En cela, nous rejoignons les conclusions d'études qui semblent établir que les aidants femmes s'ajusteraient mieux que les hommes aux troubles cognitifs et émotionnels des personnes victimes d'un AVC [31]. Les femmes retirent certes une satisfaction de l'aide prodiguée quotidiennement à autrui [32], mais est-ce le signe d'un trait constitutif du genre féminin ou plutôt une manière de réinterpréter positivement une situation [33] qu'elles ne peuvent pas changer?

Les hommes ont été nombreux à percevoir l'aide comme étant valorisante. La question qui demeure est alors : les hommes estimeraient-ils l'aide parce que la présence d'un soutien familial contribue à alléger, et donc rendre appréciables les tâches qui y sont associées ? Le fait d'aider un proche est peut-être associé pour eux au fait de remplir un "rôle protecteur ", d'où leur tendance à déclarer qu'ils prennent plaisir à prendre soin de l'autre. Gèrentils la situation comme le décrivent certaines théories sur le genre, de manière managériale [34] ? Dans ce cas, l'aide informelle serait conçue comme une tâche supplémentaire à effectuer, un nouvel objectif à atteindre.

Malgré ces observations, attribuer a priori à une personne un style d'aide selon son genre doit être évité [30].

La principale conclusion à laquelle nous aboutissons est que la survenue de l'AVC chez un proche a changé son rapport aux activités, et ce particulièrement chez les femmes: augmentation des tâches contraignantes, diminution 
d'activités divertissantes ou sociales telles que l'organisation des voyages et des invitations. Selon l'angle de la théorie du stress-coping, les femmes tendent en effet à privilégier des réponses non favorables à leur bien-être [35]. Nos réflexions nous amènent à poser l'hypothèse selon laquelle le genre pourrait contribuer à expliquer la récurrence de résultats défavorables observés chez les femmes en tant que facteur interne de la health capability [22]. Ces résultats sont à relativiser et à contextualiser en fonction du profil sociodémographique de notre population car plus de la moitié des aidantes n'ont jamais travaillé et ont un bas niveau d'instruction. La survenue de l'AVC et sa prise en charge ont sans aucun doute créé des difficultés supplémentaires dans l'accomplissement des nouvelles responsabilités qui se sont présentées à elles. De plus, elles s'occupent plus fréquemment que les hommes de cadres ou professions indépendantes qui peuvent vivre plus mal les changements survenus suite à l'AVC, quoique Badoux observe plutôt que les malades chroniques cadres présentent moins de stress que les ouvriers et personnels de service [36]. L'influence des caractéristiques sociodémographiques des malades sur les réactions émotionnelles des aidants femmes et hommes mériterait elle aussi d'être approfondie, tout comme la gravité de leurs séquelles suite à l'AVC, qui ont amené $21 \%$ de la population de notre étude à être reconnus dépendants par la Caisse Nationale de Santé luxembourgeoise. Il a par ailleurs été observé que les aidants informels de patients AVC masculins rapportent une satisfaction inférieure à l'égard de la vie [6] et sont plus souvent dépressifs [12] que les aidants de victimes d'AVC féminines. Cette observation n'est pas exclusive à l'AVC puisqu'une détresse plus importante est également observée chez les aidants de patients schizophrènes masculins [37].

\section{Limites}

Notre taux de participation estimé est de $26,5 \%$, et donc similaire à celui d'autres études (27\%) [38]. L'envoi des demandes de consentement par voie postale réduit inévitablement le taux de réponses. Il est possible qu'un certain nombre de personnes n'aient également pas souhaité recevoir un(e) enquêteur(trice) à domicile. Les répondants présentent donc des caractéristiques spécifiques.

La méthode du face à face, retenue par rapport à un questionnaire autoadministré en raison du profil de la population ciblée (10\% des personnes interrogées sont âgés d'au moins 80 ans, plusieurs langues sont parlées au Luxembourg) a pu susciter des réponses biaisées par la désirabilité sociale des répondants. À l'opposé, elle a permis d'ouvrir un lieu de parole avec une personne neutre pour exprimer leurs affects et leurs besoins, tendance qui semble être plutôt apparue parmi les aidants femmes.

\section{Perspectives}

Les principaux résultats de notre étude sur les différences de genre observées dans la manière de réagir au quotidien sont certainement le reflet de l'existence d'une spécificité dans les ressources des aidants informels hommes et femmes. Plutôt que de se focaliser sur les problèmes rencontrés par les aidants, une perspective intéressante à approfondir est de se concentrer sur leurs capacités et leurs ressources [39]. La recherche des 
déterminants de l'estime de l'aide, issue du CRA, permet de mieux saisir l'importance du rôle des facteurs psychologiques et sociaux prédisposant, contribuant ou encore renforçant le maintien ou le développement chez les aidants informels d'un accompagnement bénéfique aux victimes d'un AVC.

\section{Implications pratiques}

Les ressources, besoins et manques des aidants informels sont à prendre en compte en dépassant les représentations collectives et les stéréotypes liés au genre [30]. Dans notre étude comme dans la plupart des travaux cités, les aidantes informelles semblent plus affectées que les hommes en termes d'organisation du temps et des tâches quotidiennes ainsi que sur leur état de santé. Si des services de « répit » n'existent pas au Luxembourg pour soulager les aidants informels de victimes d'AVC, un suivi de type counseling peut leur être proposé dès le retour à domicile pour détecter les aidants les plus en souffrance et les aider à surmonter leurs problèmes. Cette technique présente l'avantage de traiter chaque situation de manière individuelle et est accessible aux personnes les moins favorisées (faible niveau d'études, détériorations liées à l'âge). Les associations de malades et d'aidants, par l'implication des membres et leur propre vécu de l'après AVC, sont également des partenaires précieux pour les aidants en difficulté. En raison du faible nombre de malades au Luxembourg, ce type de soutien est encore peu développé dans ce pays. En revanche, il existe en Europe des initiatives intéressantes. Par exemple, la mise en place de trialogues [40], permet, par des rencontres régulières entre malades, aidants informels et professionnels de santé, de confronter les perceptions de représentants de ces trois entités les plus directement concernées par les répercussions de l'AVC. Les éventuels désaccords entre subjectivité individuelle, rapports interpersonnels et contraintes économiques et environnementales ont la possibilité d'être verbalisés et surmontés. Les stéréotypes liés au genre sont ainsi dépassés puisque les personnes parlent en leur nom propre, le discours étant ancré dans le vécu individuel. De plus, à l'image du monde associatif, l'insertion dans ce type de réunions ouvertes évite aux aidants l'isolement social.

Aucun conflit d'intérêt déclaré

\section{REMERCIEMENTS}

Ce projet est financé par le Fonds National de la Recherche (FNR) du Luxembourg. La base de données utilisée est issue du projet FNR/VIVRE/06/06/06 «Vivre au Grand-Duché de Luxembourg après un accident vasculaire cérébral : Retentissement familial et qualité de vie. Équité d'accès aux soins et aux ressources sociales ").

\section{BIBLIOGRAPHIE}

1. Eurostat. Health statistics - Atlas on mortality in the European Union [Internet]. Office for Official Publications of the European Communities. Luxembourg: 2009 [cited 2011 Aug 12]. 215 p. Available from: http://epp.eurostat.ec.europa.eu/cache/ITY_OFFPUB/KS-30-08-357/EN/KS-30-08-357-EN.PDF

2. Baumann M, Lurbe K, Leandro ME, Chau N. Life satisfaction of two-year post-stroke survivors: effects of socio-economic factors, motor impairment Newcastle stroke-specific quality of life measure and world health organization quality of life - Bref of informal caregivers in Luxembourg and a rural area in Portugal. Cerebrovasc Dis. 2012 ;33:219-30. 
3. Antoine $\mathrm{P}, \mathrm{Quandalle} \mathrm{S}$, Christophe V. Vivre avec un proche malade : évaluation des dimensions positive et négative de l'expérience des aidants naturels. Ann Med Psychol. revue psychiatrique. 2010 mai; 168(4):273-82.

4. Baumann M, Aïach P. L'« aidant principal » face à l'AVC d'un proche. Médecine. 2009;5:184-8.

5. Montgomery RJV, Gonyea JG, Hooyman NR. Caregiving and the experience of subjective and objective burden. Fam relat. 1985:19-26.

6. Forsberg-Wärleby G, Möller A, Blomstrand C. Life satisfaction in spouses of patients with stroke during the first year after stroke. J Rehabil Med. 2004 Jan; 36(1):4-11.

7. Draper $P$, Brocklehurst $\mathrm{H}$. The impact of stroke on the well-being of the patient's spouse: an exploratory study. J Clin Nurs. 2007 Feb;16(2):264-71.

8. Smith LN, Norrie J, Kerr SM, Lawrence IM, Langhorne P, Lees KR. Impact and influences on caregiver outcomes at one year post-stroke. Cerebrovasc. Dis. 2004; 18(2):145-53.

9. Dennis M, O’Rourke S, Lewis S, Sharpe M, Warlow C. A quantitative study of the emotional outcome of people caring for stroke survivors. Stroke. 1998 Sep; 29(9):1867-72.

10. McCarthy MJ, Powers LE, Lyons KS. Poststroke depression: social workers' role in addressing an underrecognized psychological problem for couples who have experienced stroke. Health Soc Work. 2011 May; 36(2):139-48.

11. Navaie-Waliser M, Feldman PH, Gould DA, Levine C, Kuerbis AN, Donelan K. When the caregiver needs care: the plight of vulnerable caregivers. Am J Public Health. 2002 Mar; 92(3):409-13.

12. Berg A, Palomäki H, Lönnqvist J, Lehtihalmes $M$, Kaste $M$. Depression among caregivers of stroke survivors. Stroke. 2005 Mar;36(3):639-43.

13. Clark MS, Smith DS. Changes in family functioning for stroke rehabilitation patients and their families. Int J Rehabil Res. 1999; 22(3):171.

14. MacRae HM. Women and Caring: Constructing Self Through Others. J Women Aging. 1995;7(1/2).

15. Panel national des aidants familiaux BVA - Fondation Novartis. Les aidants familiaux en France [Internet]. 2010 [consulté le 1 er août 2011] accessible: http://www.fedesap.org/v2/documents/AidantsFamiliaux_EtudeBVA_Novartis.pdf

16. Grant JS, Weaver M, Elliott TR, Bartolucci AR, Newman Giger J. Sociodemographic, physical and psychosocial factors associated with depressive behaviour in family caregivers of stroke survivors in the acute care phase. Brain Inj. 2004 Aug;18(8):797-809.

17. Navaie-Waliser M, Spriggs A, Feldman PH. Informal caregiving: differential experiences by gender. Med Care. 2002 Dec;40(12):1249-59.

18. Green TL, King KM. Experiences of male patients and wife-caregivers in the first year post-discharge following minor stroke: a descriptive qualitative study. Int J Nurs Stud. 2009 Sep;46(9):1194-200.

19. McCullagh E, Brigstocke G, Donaldson N, Kalra L. Determinants of caregiving burden and quality of life in caregivers of stroke patients. Stroke. 2005 Oct;36(10):2181-6.

20. Calasanti T, Bowen ME. Spousal caregiving and crossing gender boundaries: Maintaining gendered identities. J Aging Stud. 2006 Sep;20(3):253-63.

21. Pierce LL, Steiner V. What are male caregivers talking about? Top Stroke Rehabil. 2004;11(2):77-83.

22. Ruger JP. Health capability: conceptualization and operationalization. Am J Public Health. 2010 Jan;100(1):41-9.

23. Nir Z, Greenberger C, Bachner YG. Profile, burden, and quality of life of Israeli stroke survivor caregivers: a longitudinal study. J Neurosci Nurs. 2009 Apr;41(2):92-105.

24. Kjellström T, Norrving B, Shatchkute A. Helsingborg Declaration 2006 on European stroke strategies. Cerebrovasc Dis. 2007;23(2-3):231-41.

25. Kelly-Hayes M, Robertson JT, Broderick JP, Duncan PW, Hershey LA, Roth EJ et al. The American Heart Association Stroke Outcome Classification. Stroke. 1998 Jun;29(6):1274-80.

26. Given CW, Given B, Stommel M, Collins C, King S, Franklin S. The caregiver reaction assessment (CRA) for caregivers to persons with chronic physical and mental impairments. Res Nurs Health. 1992 Aug;15(4):271-83.

27. Forsberg-Wärleby G, Möller A, Blomstrand C. Psychological well-being of spouses of stroke patients during the first year after stroke. Clin Rehabil. 2004 Jun; 18(4):430-7.

28. Allen SM. Gender differences in spousal caregiving and unmet need for care. J Gerontol. 1994; 49(4):187-95.

29. Pinquart M, Sörensen S. Gender differences in caregiver stressors, social resources, and health: an updated meta-analysis. J Gerontol B Psychol Sci Soc Sci. 2006; 61(1):33-45.

30. Carroll M, Campbell L. Who now reads Parsons and Bales: Casting a critical eye on the "gendered styles of caregiving" literature. J Aging Stud. 2008 Jan; 22(1):24-31.

31. Alexander T, Wilz G. Family caregivers: gender differences in adjustment to stroke survivors' mental changes. Rehabil Psychol. 2010 May;55(2):159-69.

32. Conner KA. Continuing to care: older Americans and their families. Falmer Press; 2000. 292 p. 
33. Carver CS. You want to measure coping but your protocol's too long: consider the brief COPE. Int J Behav Med. 1997;4(1):92-100.

34. Harris PB. The voices of husbands and sons caring for a family member with dementia. In: Men as Caregivers: Theory, Research and Service Implications. Springer Publishing Company; 2002. p. 416.

35. Lutzky SM, Knight BG. Explaining gender differences in caregiver distress: The roles of emotional attentiveness and coping styles. Psychol Aging. 1994;9(4):513.

36. Badoux A. Facteurs de stress et maladies chroniques. Cahiers d'études et de recherches francophones/ Santé. 2000; 10(5):345-51.

37. Sibitz I, Amering M, Kramer B, Griengl H, Katschnig H. [The course of illness and problems of schizophrenic women and men from the relatives' perspective]. Psychiatr Prax. 2002 Apr;29(3):148-53.

38. Bergström AL, Eriksson G, von Koch L, Tham K. Combined life satisfaction of persons with stroke and their caregivers: associations with caregiver burden and the impact of stroke. Health Qual Life Outcomes. 2011;9:1.

39. Peacock S, Forbes D, Markle-Reid M, Hawranik P, Morgan D, Jansen L et al. The Positive Aspects of the Caregiving Journey With Dementia: Using a Strengths-Based Perspective to Reveal Opportunities. J Appl Gerontol. 2010;29(5):640.

40. Amering M. Trialog-an exercise in communication between consumers, carers and professional mental health workers beyond role stereotypes. Int J Integr Care. 2010;10 Suppl:e014. 